\title{
Deep learning-based grading of ductal carcinoma in situ in breast histopathology images
}

\author{
Suzanne C. Wetstein $\mathbb{1}^{1} \cdot$ Nikolas Stathonikos $\mathbb{D}^{2} \cdot$ Josien P. W. Pluim ${ }^{1} \cdot$ Yujing J. Heng $\mathbb{D}^{3} \cdot$ Natalie D. ter Hoeve ${ }^{2} \cdot$ \\ Celien P. H. Vreuls ${ }^{2}$ Paul J. van Diest ${ }^{2} \cdot$ Mitko Veta $^{1}$
}

Received: 13 October 2020 / Revised: 5 January 2021 / Accepted: 7 January 2021 / Published online: 19 February 2021

(c) The Author(s) 2021. This article is published with open access

\begin{abstract}
Ductal carcinoma in situ (DCIS) is a non-invasive breast cancer that can progress into invasive ductal carcinoma (IDC). Studies suggest DCIS is often overtreated since a considerable part of DCIS lesions may never progress into IDC. Lower grade lesions have a lower progression speed and risk, possibly allowing treatment de-escalation. However, studies show significant inter-observer variation in DCIS grading. Automated image analysis may provide an objective solution to address high subjectivity of DCIS grading by pathologists. In this study, we developed and evaluated a deep learning-based DCIS grading system. The system was developed using the consensus DCIS grade of three expert observers on a dataset of 1186 DCIS lesions from 59 patients. The inter-observer agreement, measured by quadratic weighted Cohen's kappa, was used to evaluate the system and compare its performance to that of expert observers. We present an analysis of the lesion-level and patient-level inter-observer agreement on an independent test set of 1001 lesions from 50 patients. The deep learning system (dl) achieved on average slightly higher inter-observer agreement to the three observers (o1, o2 and o3) $\left(\kappa_{01, \mathrm{dl}}=0.81\right.$, $\kappa_{02, \mathrm{dl}}=0.53$ and $\left.\kappa_{03, \mathrm{dl}}=0.40\right)$ than the observers amongst each other $\left(\kappa_{01,02}=0.58, \kappa_{01,03}=0.50\right.$ and $\left.\kappa_{02,03}=0.42\right)$ at the lesion-level. At the patient-level, the deep learning system achieved similar agreement to the observers $\left(\kappa_{01, \mathrm{dl}}=0.77, \kappa_{\mathrm{o} 2, \mathrm{dl}}=\right.$ 0.75 and $\left.\kappa_{03, \mathrm{dl}}=0.70\right)$ as the observers amongst each other $\left(\kappa_{01,02}=0.77, \kappa_{\mathrm{o} 1,03}=0.75\right.$ and $\left.\kappa_{02,03}=0.72\right)$. The deep learning system better reflected the grading spectrum of DCIS than two of the observers. In conclusion, we developed a deep learning-based DCIS grading system that achieved a performance similar to expert observers. To the best of our knowledge, this is the first automated system for the grading of DCIS that could assist pathologists by providing robust and reproducible second opinions on DCIS grade.
\end{abstract}

Supplementary information The online version contains supplementary material available at https://doi.org/10.1038/s41374021-00540-6.

Suzanne C. Wetstein

s.c.wetstein@tue.nl

1 Medical Image Analysis Group, Department of Biomedical Engineering, Eindhoven University of Technology, Eindhoven, The Netherlands

2 Department of Pathology, University Medical Center Utrecht, University Utrecht, Utrecht, The Netherlands

3 Department of Pathology, Harvard Medical School, Beth Israel Deaconess Medical Center, Boston, MA, USA

\section{Introduction}

Breast cancer remains one of the leading causes of death in women [1]. Most breast cancers are invasive ductal carcinomas (IDCs) which arise from epithelial cells lining the ducts. Ductal carcinoma in situ (DCIS) refers to the pre-invasive stage whereby the cancer cells remain contained within the basement membrane. Studies suggest that a considerable part of DCIS lesions may never progress into IDC [2-4]. Autopsy studies indicate that occult DCIS exists in 9\% (range 0-15\%) of women [5]. A few small-scale studies have been done on patients where misdiagnosis of DCIS led to the omission of surgery. In the course of 30 years, $14-53 \%$ of these patients developed IDC [6-8]. A meta-analysis of multiple studies of patients with DCIS showed a 15 -year invasive local recurrence rate of $28 \%$ after a diagnosis of DCIS on excisional biopsy [9]. Thus, there is a substantial portion of DCIS lesions that may never develop into IDC. 
Since it is challenging to predict which patients will and will not progress to IDC [10], the diagnosis of DCIS prompts immediate surgical treatment. This decision is currently made regardless of the histologic grade of the lesion, while lower grade lesions have a lower progression speed and risk [11]. High-grade DCIS cases represent $42-53 \%$ of total cases [12-15] and are considered to have a high risk for recurrence [14, 16-18] and breast cancerspecific mortality [19].

In view of the perceived need to de-escalate treatment of DCIS, ongoing clinical trials (LORD [2], LORIS [3], COMET [20, 21] and LARRIKIN [22]) aim to monitor disease progression of patients with low risk DCIS (based on the histologic grade of their DCIS lesions) that forgo surgical treatment. As such, accurate histologic grading of DCIS is crucial for the clinical management of these patients. The aforementioned clinical trials utilize different classification systems to grade DCIS lesions as good (grade 1), moderate (grade 2) or poor (grade 3) differentiation. Histologic grading systems commonly used in practice are the Van Nuys classification [23], Holland classification [24], and Lagios classification [25]. Schuh et al. [26] compared grading among 13 pathologists using these three grading systems and 43 DCIS cases. They found that all systems had at best moderate agreement, the best being the Van Nuys classification $(\kappa=0.37)$. Other studies also showed significant inter-observer variation in DCIS grading, regardless of the grading system used [27-32].

The subjectivity and low reproducibility of histologic DCIS grading make it amenable for automated assessment by image analysis. Automated systems have the potential to decrease the workload of pathologists and standardize clinical practice $[33,34]$. Deep learning-based grading and survival prediction have been previously applied to histopathology images [34-38] and deep neural network models have been successfully developed for other tasks specific to breast histopathology [39-46]. Bejnordi et al. [47] designed a successful DCIS detection algorithm that works fully automatically on whole slide images (WSIs). The system detects epithelial regions in WSIs and classifies them as DCIS or benign/normal (i.e., malignant tissue was not a part of this study). Eighty percent of DCIS lesions were detected, at an average of 2.0 false positives per WSI. This system only detects DCIS lesions and does not grade them.

In this paper we describe the development of an automated deep learning DCIS grading system. Our unique system was developed using consensus grades based on grading by three expert observers and also incorporates the uncertainty in DCIS grading between these expert observers. In an independent test set, we compared the DCIS grading results at both lesion- and patient-level between our deep learning system and three expert observers.

\section{Materials and methods}

\section{Study design and population}

Digital slides were retrieved from the digital pathology archive of the University Medical Center in Utrecht, The Netherlands, from cases dated between Jan 1, 2016 and Dec 31 , 2017, for patients who underwent a breast biopsy or excision and were labeled with 'ductal carcinoma in situ'. This included all cases that contained DCIS regardless of the main diagnosis (i.e., cases with IDC and DCIS were also included). Since images were used anonymously, informed consent was not needed. For each patient up to three representative hematoxylin and eosin (H\&E) stained WSIs containing DCIS lesions were selected by expert observers. In total, 116 WSIs from 109 patients were included in this study. The slides were scanned using the Nanozoomer 2.0XR (Hamamatsu Phonics Europe GmbH, CJ Almere, The Netherlands) at $\times 40$ magnification with a resolution of $0.22 \mu \mathrm{m}$ per pixel.

\section{Pathological assessment}

Histologic grading into grades 1, 2 or 3 was performed according to the Holland classification system [24]. This classification system is recommended by The Netherlands Comprehensive Cancer Organisation [48] and focuses on nuclear morphologic and architectural features. Low grade nuclei have a monotonous appearance and a small size not much larger than normal epithelial cell size. Nucleoli and mitoses only occur occasionally. In contrast, high grade nuclei show marked pleomorphism, are large in size and contain one or more conspicuous nucleoli. Intermediate grade nuclei are defined as neither low nor high grade [49]. Architecturally, low grade DCIS is cribriform and/or micropapillary, while high grade DCIS is solid and often shows central necrosis.

All DCIS lesions present in the 116 WSIs were annotated by two experienced pathologists and one pathology assistant who grades cases on a regular basis. This was done using the open-source software Automated Slide Analysis Platform (ASAP; Computation Pathology Group, Radboud University Medical Center, Nijmegen, The Netherlands). Each DCIS lesion was outlined by one observer and, if necessary, the diagnosis of DCIS was confirmed with immunohistochemical staining. All outlined lesions were independently graded by all three observers. In total, 2187 lesions were annotated. A consensus grade for each DCIS lesion was obtained by majority voting. In the case where all three observers gave a different grade the assigned consensus grade was grade 2 . For the expert observers, the DCIS grade at the patient-level was assigned as the highest 
lesion grade present for the respective patient, although patients can have heterogeneous lesions [50].

\section{Development of the deep learning system}

For the development and validation of the deep learning system the 109 patients were randomly assigned to three distinct subsets: training, validation (used for model selection and parameter tuning) and test datasets, whilst ensuring the distribution of DCIS grades was similar in each subset. The training set contained 879 DCIS lesions from 40 patients, the validation set contained 307 lesions from 19 patients and the test set contained 1001 lesions from 50 patients.

The data acquisition process resulted in WSI with outlined DCIS lesions. The DCIS lesions were extracted from the WSI by fitting a rectangular box around the manually annotated lesions. An additional $90 \mu \mathrm{m}$ border was drawn around these boxes in order to include the DCIS lesion as well as the surrounding stroma. The stroma was included because tumor-associated stroma has been shown to be detected in greater amounts around DCIS grade 3 than DCIS grade 1 [42] and DCIS associated stromal changes might play a role in progression to IDC [51]. The boxes were extracted at magnification level $\times 10$.

The deep learning system developed to grade DCIS takes into account the inter-observer variability in DCIS grading. The system was trained on two targets: (1) the consensus of the DCIS grades given by the three expert observers, and (2) the number of observers that agreed with this consensus grade. This was done as we believe there can be extra information in the inter-observer variability in DCIS annotations. A lesion that was annotated as grade 1 by two observers and as grade 2 by the third observer is probably a borderline case, while a lesion that was annotated as grade 1 by all three observers is more clear-cut. By giving the system the information which cases in the training set are borderline cases it might be able to learn the distinction between the grades better. To evaluate the added value of the inclusion of observer agreement we compared our deep learning system with a baseline system which was trained on the consensus DCIS grades only. Our deep learning system outperformed this baseline system on the validation set.

The deep learning system was based on the Densenet121 [52] network architecture. As input to the network we cropped a random patch of $512 \times 512$ pixels (about 450 $\mu \mathrm{m} \times 450 \mu \mathrm{m})$ from a DCIS lesion and used data augmentation to overcome the variability of the tissue staining appearance, which is an important hurdle in histopathology image analysis [53]. The deep learning system was trained on patches extracted from the training dataset. The validation dataset was used to monitor the performance of the network during training and to prevent overfitting. All further results shown in this paper will be results on the independent test set. During evaluation on the test dataset, we extracted 10 randomly located patches from one lesion and took the median of the predicted grades as the predicted grade. No data augmentation was used during test time. More details on the deep learning system and its hyperparameters can be found in Supplementary Information. The deep learning system developed in this study was made available for scientific and non-commercial use through our Github page (https://github.com/tueimage/DCIS-grading). The dataset will also be made publicly available via the grand-challenge.org platform.

As stated before, for the expert observers the DCIS grade at the patient-level was determined by the highest lesion grade present for the respective patient. Expert observers grade a case by examining all DCIS lesions in a WSI while the algorithm is only shown one lesion at a time. Examining all lesions at once might lead to grading lesions more similarly, leading to "regression to the mean". To mimic the practice of expert observers, we chose to let the automated patient-level grade be determined by the lesion at the $P$ th percentile, where the value of $P$ was determined by best patient-level DCIS grading performance on the validation dataset. For the deep learning system, this resulted in the patient-level grade being determined by the lesion at the 80th percentile.

\section{Statistical analysis}

The inter-observer and model-vs-observer agreement for the DCIS grading was measured using quadratic weighted Cohen's Kappa. This measure is commonly used for interrater agreement on an ordinal scale because it compensates for the degree of error in category assessment. This means that disagreement by one grade point is weighted less than disagreement by two grade points. Using this method, each observer was compared with every other and Kappa values were recorded for each pairing. All analyses were performed using Python version 3.6 and the deep learning model was implemented using the Keras deep learning framework [54].

\section{Results}

\section{Population characteristics}

Patient- and lesion-level characteristics for our test dataset are summarized in Table 1. Mean patient age was 58 years (95\% CI: 55-61 years) and the number of lesions per patient was 20 (95\% CI: 11-30). Using the consensus grade of the three observers, there were seven patients with DCIS 
Table 1 Patient and lesion characteristics in the test dataset.

\begin{tabular}{ll}
\hline Patient characteristics & \\
\hline$n$ & 50 \\
Age at biopsy & $58(55-61)$ \\
$\quad$ Mean years $(95 \%$ CI) & \\
Number of lesions per patient & $20(11-30)$ \\
$\quad$ Mean $n$ (95\% CI) & \\
Consensus grade $(n, \%)$ & $7(14 \%)$ \\
$\quad$ Grade 1 & $24(48 \%)$ \\
$\quad$ Grade 2 & $19(38 \%)$ \\
$\quad$ Grade 3 & 1001 \\
\hline Lesion characteristics & \\
\hline$n \quad$ Lesion size & $0.48(0.37-0.60)$ \\
$\quad$ Mean mm ${ }^{2}(95 \%$ CI) & \\
Consensus grade $(n, \%)$ & $152(15 \%)$ \\
$\quad$ Grade 1 & $645(64 \%)$ \\
Grade 2 & $204(20 \%)$ \\
Grade 3 &
\end{tabular}

grade 1, 24 patients with grade 2 and 19 patients with grade 3 . The average lesion area was $0.48 \mathrm{~mm}^{2}(95 \% \mathrm{CI}$ : $0.37-0.60 \mathrm{~mm}^{2}$ ). There were 152 grade 1 lesions, 645 grade 2 lesions and 204 grade 3 lesions.

\section{Lesion-level inter-observer agreement in test dataset}

Inter-observer agreement on DCIS grading at the lesionlevel between three expert observers and the deep learning system is shown in Table 2. Inter-observer agreement between expert observers was $\kappa=0.58, \kappa=0.50$ and $\kappa=$ 0.42 . The deep learning system showed agreement with the observers of $\kappa=0.81, \kappa=0.53$ and $\kappa=0.40$. The average agreement between expert observers was lower than that between expert observers and the deep learning system. The confusion matrices of inter-observer agreement on DCIS grading between expert observers and the deep learning system are shown in Fig. 1. Interestingly, there was high agreement between observers 2 and 3 for grade 2 (563 lesions), but both observers graded more than half of the lesions as grade 2 (observer 2: 693 out of 1001, observer 3: 749 out of 1001 lesions). In contrast, observer 1 and the deep learning system graded only 481 and 512 lesions, respectively, as grade 2 .

Ten lesions from five patients had high disagreement (i.e., cases being assigned grade 1 and grade 3 ) between two expert observers or between an expert observer and the deep learning system (Fig. 2). These lesions were all very small with an area $\leq 0.06 \mathrm{~mm}^{2}$, while the average
Table 2 Inter-observer quadratic weighted Cohen's Kappa for ductal carcinoma in situ (DCIS) grading at the lesion-level among three observers and the deep learning system.

\begin{tabular}{clcl}
\hline & \multicolumn{2}{c}{ Expert observers } & \multirow{2}{*}{$\begin{array}{l}\text { Deep learning } \\
\text { system }\end{array}$} \\
\cline { 2 - 3 } & Observer 2 & Observer 3 & \\
\hline & $\kappa(95 \% \mathrm{CI})$ & $\kappa(95 \% \mathrm{CI})$ & $\kappa(95 \% \mathrm{CI})$ \\
Observer 1 & $0.58(0.49-0.66)$ & $0.50(0.41-0.60)$ & $0.81(0.75-0.86)$ \\
Observer 2 & - & $0.42(0.29-0.54)$ & $0.53(0.44-0.62)$ \\
Observer 3 & - & - & $0.40(0.29-0.54)$ \\
\hline
\end{tabular}

The results are shown on the test set which contains 1001 lesions from 50 different patients. The $95 \%$ confidence interval (CI) was determined analytically.

lesion area was $0.48 \mathrm{~mm}^{2}\left(95 \% \mathrm{CI}: \quad 0.37-0.60 \mathrm{~mm}^{2}\right)$. Upon review of the lesions, by a consensus meeting of the three expert observers, two lesions concerned small isolated detachments (floaters) and five were potentially incorrectly annotated as DCIS. For the remaining three lesions, the deep learning system classified two correctly (the high disagreement was caused by one of the expert observers) and one incorrectly.

\section{Patient-level inter-observer agreement in test dataset}

Inter-observer agreement between three expert observers and the deep learning system on DCIS grading at the patient-level is shown in Table 3. Inter-observer agreement between expert observers was $\kappa=0.77, \kappa=0.75$ and $\kappa=$ 0.72 . The deep learning system showed agreement with the observers of $\kappa=0.77, \kappa=0.75$ and $\kappa=0.70$. The average agreement between expert observers was slightly higher than that between expert observers and the deep learning system. The confusion matrices for DCIS grading at the patient-level by expert observers and our deep learning system are shown in Fig. 3. At the patient level, there was no large disagreement (i.e., no case assigned as grade 1 and grade 3 ) between two expert observers or between an expert observer and the deep learning system.

\section{Discussion}

DCIS currently prompts immediate surgical treatment while a considerable part of DCIS lesions may never progress into IDC. Lower grade lesions progress into IDC less often and at a slower pace. However, grading systems dividing DCIS lesions into low/middle/high grade were shown to have significant inter-observer variation. Accurate and reproducible DCIS grading may be possible with the help of automated image analysis. 

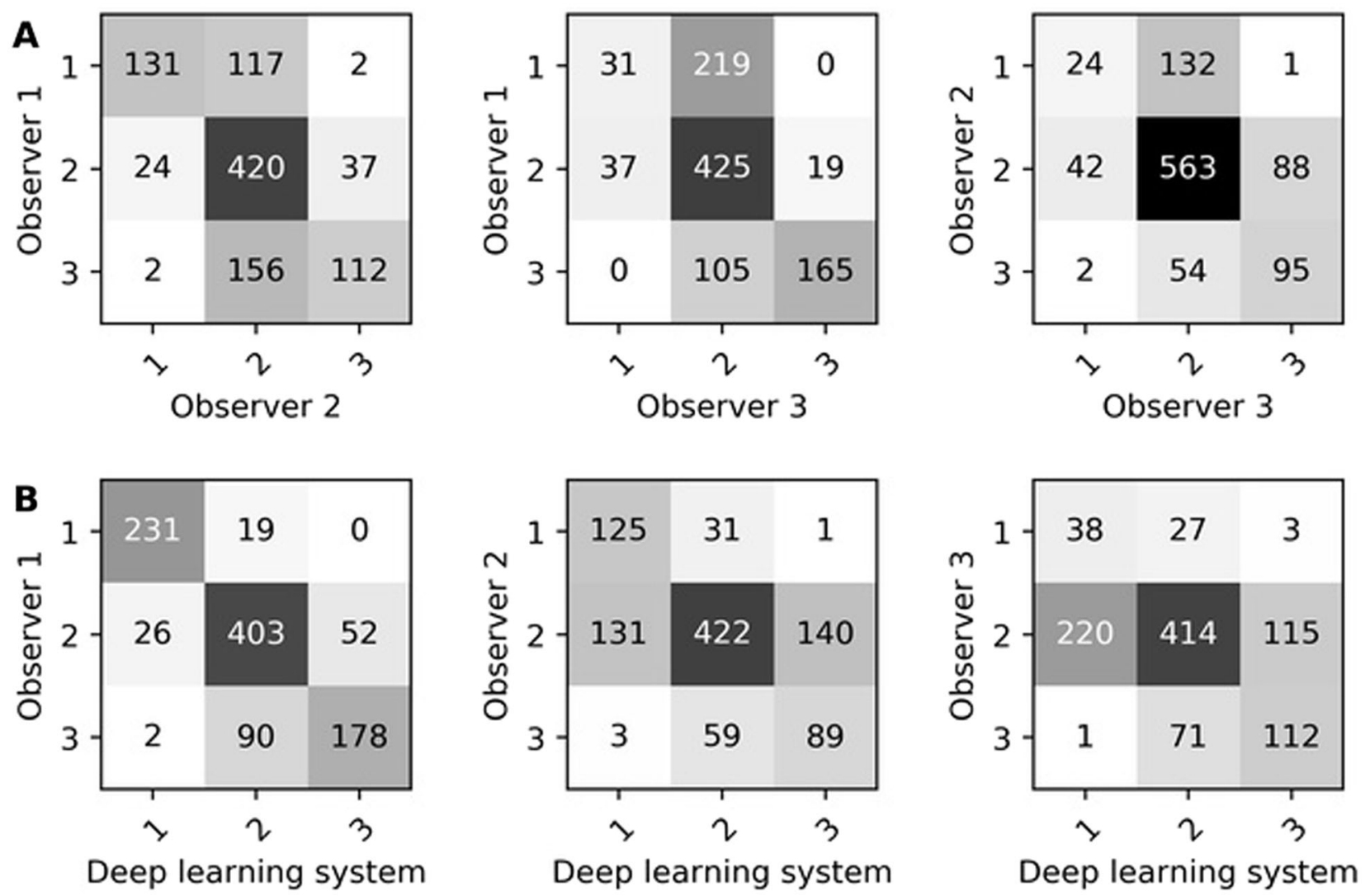

Fig. 1 Confusion matrices for DCIS grading at the lesion-level. Confusion matrices for DCIS grading at the lesion-level between observers (A) and between observers and the deep learning system (B). These are results on the test set which contained 1001 DCIS lesions from 50 patients.

We developed a fully automated deep learning system to grade DCIS lesions of the breast. To the best of our knowledge, this is the first automated classification system for the grading of DCIS. Our study demonstrated that our automated system achieved a performance similar to expert observers. The system has the potential to improve DCIS grading by acting as a reliable and consistent first or second reader.

In this study, three observers achieved an average quadratic weighted Kappa score at the lesion-level of 0.50. This is similar to Douglas-Jones et al. [30] who found an average quadratic weighted Kappa score of 0.48 between 19 pathologists for Van Nuys classification of 60 DCIS lesions. Other studies have also shown high inter-observer variability for DCIS grading but used other measures of agreement, like unweighted Cohen's Kappa or percentage of agreement between observers [26-29, 31, 32]. The high inter-observer variability between observers reiterates the need to create a consistent system for DCIS grading.

We developed a deep learning system to grade DCIS that incorporates the inter-observer variability (aleatoric uncertainty) in the training process. At the lesion-level, the system achieved agreement with observers comparable to observers amongst each other. The confusion matrices
(Fig. 1) showed high agreement between observer 1 and the deep learning system. These matrices also showed that observers 2 and 3 graded many cases $(69 \%$ and $75 \%$, respectively) as grade 2 . In contrast, observer 1 and the deep learning system graded only $48 \%$ and $51 \%$ of lesions, respectively, as grade 2 . Therefore, grading by both observer 1 and the deep learning system was more diversified, better reflecting the grading spectrum of DCIS.

We found high disagreement (i.e., cases being assigned grade 1 and grade 3) between observers and between observers and the deep learning system in ten lesions as shown in Fig. 2. Re-examination of these lesions showed that two lesions concerned small isolated detachments (floaters) and five were potentially incorrectly annotated as DCIS. Since these errors only occurred in 7 out of 1001 lesions (i.e., $0.7 \%$ ), we decided neither to exclude the floaters and wrongly annotated lesions from the dataset nor rerun the analysis as it would unlikely yield significantly better results. For the remaining three lesions, the deep learning system classified two correctly (one of the expert observers caused the high disagreement) and one incorrectly. All of these lesions were very small (area $\leq 0.06 \mathrm{~mm}^{2}$ ). We hypothesize that both expert observers and deep learning have difficulties classifying tiny 

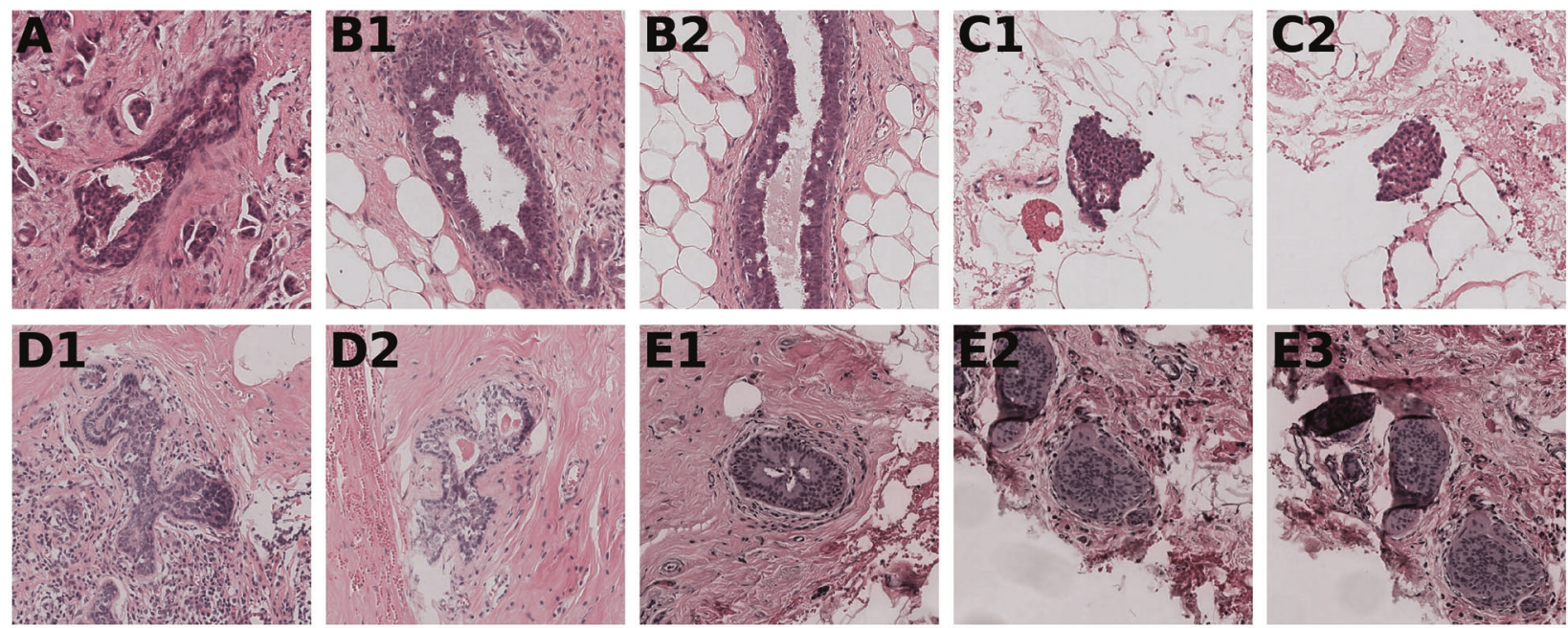

Fig. 2 All lesions with high disagreement between expert observers and between expert observers and the deep learning system. Lesions with the same letter come from the same patient. All lesions had an image size of $512 \times 512$ pixels except for (B2) where we show the middle $512 \times 512$ pixel patch. For lesion (A) the observers graded 2-3-2 and the deep learning system predicted grade 1 . On final review in a consensus meeting, grades 1 and 3 did not seem justified, therefore the expert observers assigned this lesion as grade 2. For lesions (B1) and (B2) the observers graded 1-3-1 and the deep learning system predicted grade 1 . Grade 3 did not seem justified during the consensus meeting and was an error by an expert observer. For lesion (C1) the

Table 3 Inter-observer quadratic weighted Cohen's Kappa for ductal carcinoma in situ (DCIS) grading at the patient-level amongst three observers and the deep learning system.

\begin{tabular}{clcl}
\hline & \multicolumn{2}{c}{ Expert observers } & \multirow{2}{*}{$\begin{array}{l}\text { Deep learning } \\
\text { system }\end{array}$} \\
\cline { 2 - 3 } & Observer 2 & Observer 3 & \\
\hline & $\kappa(95 \% \mathrm{CI})$ & $\kappa(95 \% \mathrm{CI})$ & $\kappa(95 \% \mathrm{CI})$ \\
Observer 1 & $0.77(0.49-1.05)$ & $0.72(0.40-1.04)$ & $0.77(0.49-1.05)$ \\
Observer 2 & - & $0.75(0.46-1.04)$ & $0.70(0.41-1.00)$ \\
Observer 3 & - & - & $0.75(0.46-1.04)$ \\
\hline
\end{tabular}

The results are shown on the test set which contains 50 patients. The $95 \%$ confidence interval (CI) was determined analytically.

lesions because there is less information to work with, and observers may have graded these lesions not so much on their specific morphologic appearance but similar to the larger surrounding ones, leading to "regression to the mean".

At the patient-level, the expert observers were slightly more in agreement with each other than with the deep learning system. The confusion matrices (Fig. 3) show that there were no grade 1 vs. grade 3 discrepancies between expert observers nor between expert observers and the deep learning system. Expert observers amongst each other agreed more on grade 3, whereas expert observers and the deep learning system agreed more on grade 1. observers graded 3-2-2 and the deep learning system predicted grade 1. For lesion (C2) the observers graded 3-2-3 and the deep learning system predicted grade 1 . Both these lesions concern floaters and should not have been in the dataset. For lesion (D1) the observers graded 3-1-2 and the deep learning system predicted grade 2. For lesion (D2) the observers graded 3-1-3 and the deep learning system predicted grade 2. On review, both lesions are not obviously DCIS. For lesion (E1) the observers graded $2-1-1$ and the deep learning system predicted grade 3. For lesions (E2) and (E3) the observers graded $2-2-1$ and the deep learning system predicted grade 3 . On review, these three lesions are not obviously DCIS.

Before implementation of our system in clinical practice, some limitations must be addressed. First, the dataset used to develop our deep learning system originated from a single medical center. Although we applied data augmentation to expand our training dataset, the robustness of the system can be improved by including WSIs from different institutions obtained using different whole slide scanners and different staining protocols. Second, DCIS grading was performed based on the Holland grading system [24]. Although this system is recommended by The Netherlands Comprehensive Cancer Organization, varying systems are used around the world. For implementation in clinical practice elsewhere, the deep learning system should be trained with data annotated according to the recommended guidelines in the respective country. Third, our deep learning system solely grades DCIS lesions. The system cannot distinguish DCIS lesions from benign lesions or IDC. In future research, we do aim to develop a system that can outline DCIS lesions and make this distinction. Combining this potential system and our current system could lead to automated DCIS grading on WSIs.

Our system was able to achieve a performance similar to that of expert observers. However, the agreement between expert observers for this task, especially at the lesion level, was not high. Due to the fact that the system was trained and tested on data annotated by expert observers it would be hard to exceed their performance. Therefore, in future 

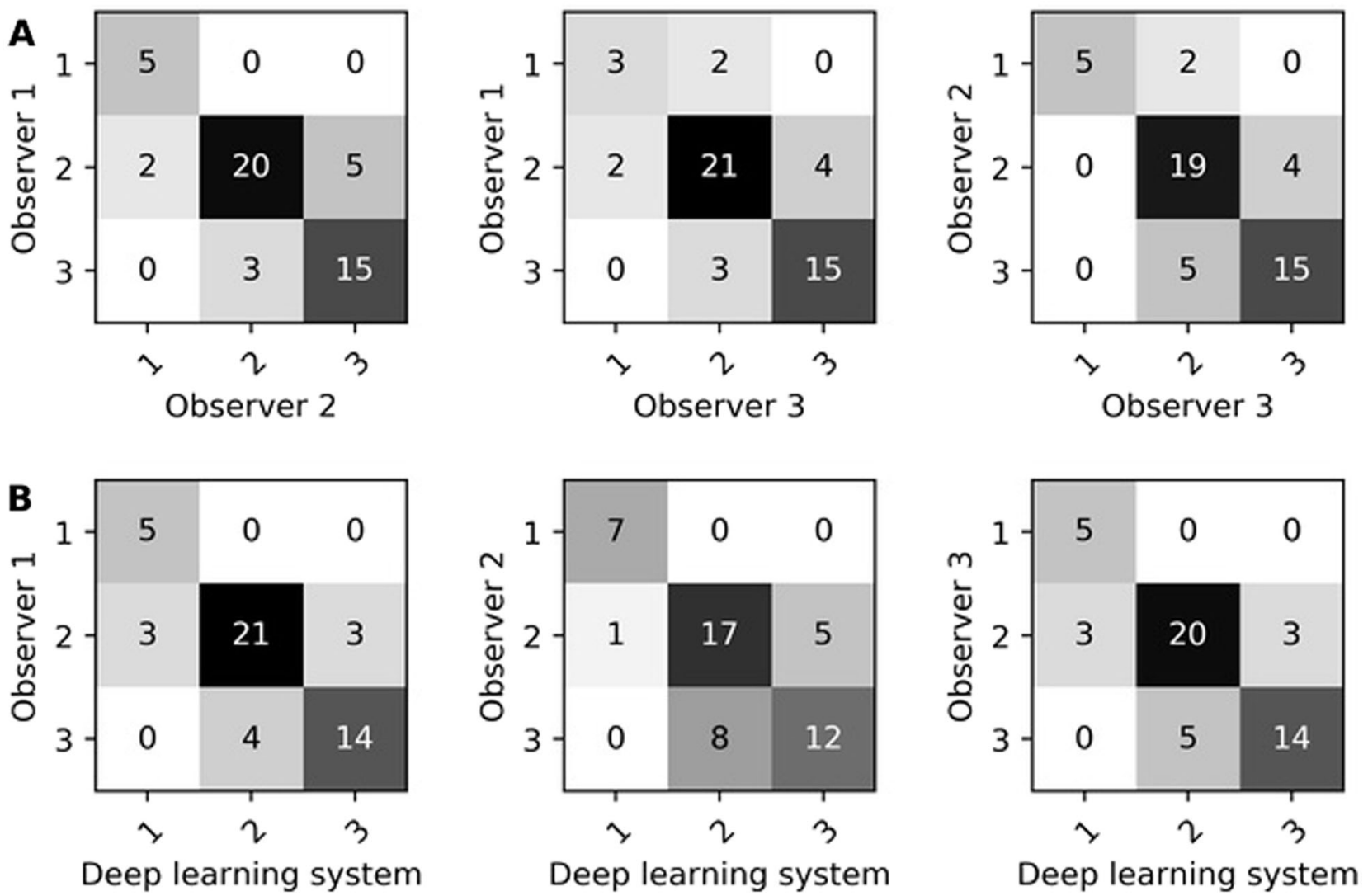

Fig. 3 Confusion matrices for DCIS grading at the patient-level. Confusion matrices for DCIS grading at the patient-level between observers (A) and between observers and the deep learning system (B). These results are on the test set which contained 50 patients.

studies it would be interesting to gather information on whether patients with DCIS progressed to IDC. This information could be used to train a deep learning system to predict which DCIS lesions have a high chance of progressing to IDC. Realistically, it would only be possible to gather follow up information for patients with low grade DCIS as it would not be safe to forgo treatment for patients with high grade DCIS. Data could possibly be gathered from low grade DCIS patients that entered clinical trials (e.g., LORD [2], LORIS [3], COMET [20, 21] and LARRIKIN [22]). The information on which low grade DCIS lesions progress to IDC within 5-10 years could be used to improve the grading practice of pathologists and automated systems.

In conclusion, we developed and evaluated an automated deep learning-based DCIS grading system which achieved a performance similar to expert observers. With further evaluation, this system could assist pathologists by providing robust and reproducible second opinions on DCIS grade.

Acknowledgements This work was supported by the Deep Learning for Medical Image Analysis research program by The Dutch Research Council P15-26 and Philips Research (SCW, MV and JPWP).

\section{Compliance with ethical standards}

Conflict of interest The authors declare no competing interests.

Publisher's note Springer Nature remains neutral with regard to jurisdictional claims in published maps and institutional affiliations.

Open Access This article is licensed under a Creative Commons Attribution 4.0 International License, which permits use, sharing, adaptation, distribution and reproduction in any medium or format, as long as you give appropriate credit to the original author(s) and the source, provide a link to the Creative Commons license, and indicate if changes were made. The images or other third party material in this article are included in the article's Creative Commons license, unless indicated otherwise in a credit line to the material. If material is not included in the article's Creative Commons license and your intended use is not permitted by statutory regulation or exceeds the permitted use, you will need to obtain permission directly from the copyright holder. To view a copy of this license, visit http://creativecommons. org/licenses/by/4.0/.

\section{References}

1. Siegel RL, Miller KD, Jemal A. Cancer statistics, 2020. CA Cancer J Clin. 2020;65:5-29.

2. Elshof LE, Tryfonidis K, Slaets L, van Leeuwen-Stok AE, Skinner VP, Dif N, et al. Feasibility of a prospective, randomised, open-label, international multicentre, phase III, non-inferiority 
trial to assess the safety of active surveillance for low risk ductal carcinoma in situ - the LORD study. EUR $J$ Cancer. 2015;51:1497-510.

3. Francis A, Thomas J, Fallowfield L, Wallis M, Bartlett JM, Brookes $\mathrm{C}$, et al. Addressing overtreatment of screen detected DCIS; the LORIS trial. Eur J Cancer. 2015;51:2296-303.

4. Benson JR, Jatoi I, Toi M. Treatment of low-risk ductal carcinoma in situ: is nothing better than something? Lancet Oncol. 2016;17:442-51.

5. Welch HG, Black WC. Using autopsy series to estimate the disease "reservoir" for ductal carcinoma in situ of the breast: how much more breast cancer can we find? Ann Intern Med. 1997; 127:1023-8.

6. Collins LC, Tamimi RM, Baer HJ, Connolly JL, Colditz GA, Schnitt SJ. Outcome of patients with ductal carcinoma in situ untreated after diagnostic biopsy: results from the Nurses' Health Study. Cancer. 2005;103:1778-84.

7. Erbas B, Provenzano E, Armes J, Gertig D. The natural history of ductal carcinoma in situ of the breast: a review. Breast Cancer Res Treat. 2006;97:135-44.

8. Sanders ME, Schuyler PA, Simpson JF, Page DL, Dupont WD. Continued observation of the natural history of low-grade ductal carcinoma in situ reaffirms proclivity for local recurrence even after more than 30 years of follow-up. Modern Pathol. 2015;28:662-9.

9. Stuart KE, Houssami N, Taylor R, Hayen A, Boyages J. Longterm outcomes of ductal carcinoma in situ of the breast: a systematic review, meta-analysis and meta-regression analysis. BMC Cancer. 2015;15:890.

10. Groen EJ, Elshof LE, Visser LL, Emiel JT, Winter-Warnars HA, Lips EH, et al. Finding the balance between over-and undertreatment of ductal carcinoma in situ (DCIS). Breast. 2017;31:274-83.

11. Sanders ME, Schuyler PA, Dupont WD, Page DL. The natural history of low-grade ductal carcinoma in situ of the breast in women treated by biopsy only revealed over 30 years of long-term follow-up. Cancer. 2005;103:2481-4.

12. Elshof LE, Schmidt MK, Emiel JT, Rutgers FE, Wesseling J, Schaapveld M. Cause-specific mortality in a population-based cohort of 9799 women treated for ductal carcinoma in situ. Ann Surg. 2018;267:952.

13. Falk RS, Hofvind S, Skaane P, Haldorsen T. Second events following ductal carcinoma in situ of the breast: a register-based cohort study. Breast Cancer Res Treat. 2011;129:929.

14. Kerlikowske K, Molinaro AM, Gauthier ML, Berman HK, Waldman F, Bennington J, et al. Biomarker expression and risk of subsequent tumors after initial ductal carcinoma in situ diagnosis. J Natl Cancer I. 2010;102:627-37.

15. Worni M, Akushevich I, Greenup R, Sarma D, Ryser MD, Myers ER, et al. Trends in treatment patterns and outcomes for ductal carcinoma in situ. J Natl Cancer Inst. 2015;107:djv263.

16. Cheung S, Booth ME, Kearins O, Dodwell D. Risk of subsequent invasive breast cancer after a diagnosis of ductal carcinoma in situ (DCIS). Breast. 2014;23:807-11.

17. Rakovitch E, Nofech-Mozes S, Hanna W, Narod S, Thiruchelvam D, Saskin R, et al. HER2/neu and Ki-67 expression predict noninvasive recurrence following breast-conserving therapy for ductal carcinoma in situ. Br J Cancer. 2012;106:1160-5.

18. Wang SY, Shamliyan T, Virnig BA, Kane R. Tumor characteristics as predictors of local recurrence after treatment of ductal carcinoma in situ: a meta-analysis. Breast Cancer Res Treat. 2011;127:1-4.

19. Narod SA, Iqbal J, Giannakeas V, Sopik V, Sun P. Breast cancer mortality after a diagnosis of ductal carcinoma in situ. JAMA Oncol. 2015;1:888-96.
20. Youngwirth LM, Boughey JC, Hwang ES. Surgery versus monitoring and endocrine therapy for low-risk DCIS: the COMET trial. Bull Am Coll Surg. 2017;102:62-3.

21. Hwang ES, Hyslop T, Lynch T, Frank E, Pinto D, Basila D, et al. The COMET (Comparison of Operative versus Monitoring and Endocrine Therapy) trial: a phase III randomised controlled clinical trial for low-risk ductal carcinoma in situ (DCIS). BMJ Open. 2019;9:e026797.

22. Lippey J, Spillane A, Saunders C. Not all ductal carcinoma in situ is created equal: can we avoid surgery for low-risk ductal carcinoma in situ? ANZ J Surg. 2016;86:859-60.

23. Poller DN, Barth A, Slamon DJ, Silverstein MJ, Gierson ED, Coburn WJ, et al. Prognostic classification of breast ductal carcinoma-in-situ. Lancet. 1995;345:1154-7.

24. Holland R, Peterse JL, Millis RR, Eusebi V, Faverly D, van de Vijver MA, et al. Ductal carcinoma in situ: a proposal for a new classification system. Semin Diagn Pathol. 1994;11:167-80.

25. Sneige N, Lagios MD, Schwarting R, Colburn W, Atkinson E, Weber $\mathrm{D}$, et al. Interobserver reproducibility of the Lagios nuclear grading system for ductal carcinoma in situ. Hum Pathol. 1999;30:257-62.

26. Schuh F, Biazus JV, Resetkova E, Benfica CZ, Edelweiss MIA. Reproducibility of three classification systems of ductal carcinoma in situ of the breast using a web-based survey. Pathol Res Pract. 2010;206:705-11.

27. Schnitt SJ, Connolly JL, Tavassoli FA, Fechner RE, Kempson RL, Gelman R, et al. Interobserver reproducibility in the diagnosis of ductal proliferative breast lesions using standardized criteria. Am J Surg Pathol. 1992;16:1133-43.

28. Bethwaite P, Smith N, Delahunt B, Kenwright D. Reproducibility of new classification schemes for the pathology of ductal carcinoma in situ of the breast. J Clin Pathol. 1998;51:450-4.

29. Sloane JP, Amendoeira I, Apostolikas N, Bellocq JP, Bianchi S, Boecker W, et al. Consistency achieved by 23 European pathologists in categorizing ductal carcinoma in situ of the breast using five classifications. European commission working group on breast screening pathology. Hum Pathol. 1998;29: 1056-62.

30. Douglas-Jones AG, Morgan JM, Appleton MA, Attanoos RL, Caslin A, Champ CS, et al. Consistency in the observation of features used to classify duct carcinoma in situ (DCIS) of the breast. J Clin Pathol. 2000;53:596-602.

31. Douglas-Jones AG, Gupta SK, Attanoos RL, Morgan JM, Mansel RE. A critical appraisal of six modern classifications of ductal carcinoma in situ of the breast (DCIS): correlation with grade of associated invasive carcinoma. Histopathology. 1996;29:397-409.

32. van Dooijeweert C, van Diest PJ, Willems SM, Kuijpers CC, Overbeek LI, Deckers IA. Significant inter- and intra-laboratory variation in grading of ductal carcinoma in situ of the breast: a nationwide study of 4901 patients in the Netherlands. Breast Cancer Res Treat. 2019;174:479-88.

33. Dimitriou N, Arandjelović O, Caie PD. Deep learning for whole slide image analysis: an overview. Front Med. 2019;6:264.

34. Litjens G, Sánchez CI, Timofeeva N, Hermsen M, Nagtegaal I, Kovacs I, et al. Deep learning as a tool for increased accuracy and efficiency of histopathological diagnosis. Sci Rep. 2016;6: 26286.

35. Bulten W, Pinckaers H, van Boven H, Vink R, de Bel T, van Ginneken B, et al. Automated deep-learning system for Gleason grading of prostate cancer using biopsies: a diagnostic study. Lancet Oncol. 2020;21:233-41.

36. Ertosun MG, Rubin DL. Automated grading of gliomas using deep learning in digital pathology images: a modular approach with ensemble of convolutional neural networks. In AMIA Annu 
Symp Proc. 2015;1899-908. American Medical Informatics Association.

37. Källén H, Molin J, Heyden A, Lundström C, Åström K. Towards grading gleason score using generically trained deep convolutional neural networks. In Proceedings of the13th International Symposium on Biomedical Imaging (ISBI). 2016:1163-7. IEEE.

38. Yue X, Dimitriou N, Caie DP, Harrison JD, Arandjelovic O. Colorectal cancer outcome prediction from $H \& E$ whole slides images using machine learning and automatically inferred phenotype profiles. In Conf Bioinform Comput Biol. 2019;60:139-49.

39. Veta M, Heng YJ, Stathonikos N, Bejnordi BE, Beca F, Wollmann T, et al. Predicting breast tumor proliferation from wholeslide images: the TUPAC16 challenge. Med Image Anal. 2019;54:111-21.

40. Wetstein SC, Onken AM, Luffman C, Baker GM, Pyle ME, Kensler $\mathrm{KH}$, et al. Deep learning assessment of breast terminal duct lobular unit involution: towards automated prediction of breast cancer risk. PLoS ONE. 2020;15:e0231653.

41. Kensler KH, Liu EZ, Wetstein SC, Onken AM, Luffman CI, Baker GM, et al. Automated quantitative measures of terminal duct lobular unit involution and breast cancer risk. Cancer Epidemiol Biomarkers Prev. 2020;29:2358-68.

42. Bejnordi BE, Mullooly M, Pfeiffer RM, Fan S, Vacek PM, Weaver DL, et al. Using deep convolutional neural networks to identify and classify tumor-associated stroma in diagnostic breast biopsies. Mod Pathol. 2018;31:1502.

43. Balkenhol MCA, Tellez D, Vreuls W, Clahsen PC, Pinckaers H, Ciompi F, et al. Deep learning assisted mitotic counting for breast cancer. Lab Invest. 2019;99:1596-606.

44. Veta M, van Diest PJ, Jiwa M, Al-Janabi S, Pluim JPW. Mitosis counting in breast cancer: object-level interobserver agreement and comparison to an automatic method. PLoS ONE. 2016;11: e0161286.
45. Wang D, Khosla A, Gargeya R, Irshad H, Beck AH. Deep learning for identifying metastatic breast cancer. Preprint arXiv https://arxiv.org/abs/1606.05718.

46. Bejnordi BE, Veta M, Van Diest PJ, Van Ginneken B, Karssemeijer N, Litjes G, et al. Diagnostic assessment of deep learning algorithms for detection of lymph node metastases in women with breast cancer. JAMA. 2017;318:2199-210.

47. Bejnordi BE, Balkenhol M, Litjens G, Holland R, Bult P, Karssemeijer N, et al. Automated detection of DCIS in whole-slide H\&E stained breast histopathology images. IEEE Trans Med Imaging. 2016;35:2141-50.

48. The Netherlands Comprehensive Cancer Organisation (IKNL). Oncoline: Breast Cancer guideline. 2017. https://www.oncoline. $\mathrm{nl} /$ borstkanker.

49. Schwartz GF. Consensus conference on the classification of ductal carcinoma in situ. Hum Pathol. 1997;28:1221-5.

50. Chapman JA, Miller NA, Lickley HL, Qian J, Christens-Barry WA, Fu Y, et al. Ductal carcinoma in situ of the breast (DCIS) with heterogeneity of nuclear grade: prognostic effects of quantitative nuclear assessment. BMC Cancer. 2007;7:174.

51. Agahozo MC, Westenend PJ, van Bockstal MR, Hansum T, Giang J, Matlung SE, et al. Immune response and stromal changes in ductal carcinoma in situ of the breast are subtype dependent. Mod Pathol. 2020;33:1773-82.

52. Huang G, Liu Z, Van Der Maaten L, Weinberger KQ. Densely connected convolutional networks. In Proceedings of the 2017 IEEE Conference on Computer Vision and Pattern Recognition (CVPR). 2017:4700-8.

53. Lafarge MW, Pluim JPW, Eppenhof KA, Moeskops P, Veta M. Domain-adversarial neural networks to address the appearance variability of histopathology images. In Deep learning in medical image analysis and multimodal learning for clinical decision support. 2017: 83-91.

54. Chollet $\mathrm{F}$ et al. Keras. 2015. https://keras.io. 